\title{
Performance Analysis of GSM Networks with Intelligent Underlay-Overlay*
}

\author{
Khalid Begain ${ }^{1}$, Gergó István Rózsa ${ }^{2}$, András Pfening $^{3}$, Miklós Telek ${ }^{4 \dagger}$ \\ ${ }^{1}$ Dept. of Computing, University of Bradford BD7 1DP, Bradford, West Yorkshire, England, UK, kbegain@bradford.ac.uk \\ ${ }^{2}$ Matáv Hungarian Telecommunications Company Ltd., Budapest, Hungary, Rozsa.Istvan.Gergo@mail.pki.matav.hu \\ ${ }^{3}$ Nokia Telecommunications, Mobile Switching Budapest, Hungary, andras.pfening@ntc.nokia.com \\ ${ }^{4}$ Department of Telecommunications, Technical University of Budapest, H-1521 Budapest, Hungary, telek@ hit.bme.hu
}

\begin{abstract}
The paper presents an analytical model for a GSM-based cellular mobile network that applies the Intelligent UnderOverlay (IUO) scheme to increase the capacity by increasing the frequency reuse while maintaining the service quality. The IUO is a multi-layer cell structure that is based on dividing the frequency band into the super layer and the regular layer frequency group. The super frequencies (channels) can be used by mobile stations with good C/I (carrier/interferer) ratio, while the regular frequencies can be used over the whole cell. The use of IUO is expected to provide up to $40 \%$ gain of capacity [1]. In this paper, we study the effect of various parameters on the performance of the networks using IUO and provide practical planning support based on the analytical results. The considered parameters include network parameters like super area coverage and mobile user mobility parameters like moving mobile ratio and average mobile speed.
\end{abstract}

Keywords: Cellular mobile networks, GSM, IUO, Performance analysis.

\section{Introduction}

The demand for wireless communication grows rapidly nowadays. The capacity of cells in the existing digital cellular mobile networks, like GSM, will have to meet the increased demand. The network operators have to face the problem, how to increase the capacity of an existing network without noticable degradation of quality of service. Different solutions can be foreseen. The most obvious solution would extend the GSM band or increase the number of serving channels or frequencies in an area. However the overall available GSM spectrum is limited and is usually divided between 2 or 3 network operators leaving a spectrum of not more than $10 \mathrm{MHz}$ for each operator. Another solution is to deploy more base stations (cell splitting), or to in-

\footnotetext{
*This work has been partially supported by OTKA T 034972 .

†Correspondence author
}

troduce hierarchical structures like micro- and picocells [2], [3]. This approach works well to a certain extent, however the denser base station grid results in increased interference that limits the quality and so the capacity in terms of soft blocking. Furthermore, the additional network elements like base stations and transmission network cost quite a lot. The most promising way of capacity enhancement requires minor investment while allowing more capacity with minor or no degradation of service quality. Two methods shall be mentioned here, the frequency hopping (FH, [4]) and the intelligent underlay-overlay proposed by Nokia Telecommunications (IUO, [1]). (Recently Nokia proposed the IFH technology, which combines IUO and FH. This is out of the scope of the paper.) Both methods are similar in the sense that they allow more frequencies to be used in the existing cells, that is increase the reuse factor, introducing minor investment cost. As a result of the tighter reuse, the interference in the network will be higher. The two methods differ in how they cope with the increased interference. In FH, the established speech connection is hopping on a number of frequencies. A number of those frequencies are "clean", that is do not suffer from serious interference, while the other frequencies are interfered. Due to the averaging effect of the different coding principles, the noise brought by the interfered frequencies is eliminated. The IUO principle is to make use of the measurements done by the mobile station, MS. The MS always measures the strongest neighbors of the serving cell in order to know when to make a handover to a neighbor cell. This measurement data is used to estimate the $\mathrm{C} / \mathrm{I}$ conditions of the MS. If the estimated $\mathrm{C} / \mathrm{I}$ is good enough, the MS is assigned a (heavily reused) so-called super frequency, while if the $\mathrm{C} / \mathrm{I}$ is bad, a clean regular frequency is assigned to the MS. This way the super frequencies (channels) can be used by mobiles with good $\mathrm{C} / \mathrm{I}$ ratio, while the regular frequencies can be used over the whole cell. The frequency band is divided into two groups, a super layer and a regular layer frequencies, and a lower frequency reuse factor, thus a smaller area of coverage is assigned to the super layer. 
The origin of IUO principle was reuse-partitioning. It was presented in some papers $[5,6,7]$. [5] presented the DCA (dynamic Channel Assignment) with a greedy algorithm, and investigated 1 dimensional cellular radio system. Simulation results were reported on IUO in $[8,9,6]$. In [10], the performance of GSM network implementing IUO in combination with Frequency hopping was studied by simulation. Some further improvements on the original IUO scheme were suggested. The effect of these improvements was reported in [11]. In this paper, we introduce an analytical model of GSM network implementing IUO and provide results on the performance of the network taking into account many parameters like the coverage factor of the super layer to the whole cell, the ratio of moving mobile stations, and the speed at which they are moving. Our paper gives practically useful view on the above mentioned parameters. Network designers can directly apply the provided results to dimension their network with improved reuse of the spectrum in cellular radio systems.

With the applied analysis approach we calculate the network performance parameters by considering a large homogeneous network composed by a lot of identical cells, whose handover traffic to the neighboring cells is symmetric. This assumption on the homogeneous network with symmetric handover traffic allows us to calculate the network performance based on the analysis of a single cell. The analysis of inhomogeneous network with asymmetric handover traffic of neighboring cells, that would require a much more complex analysis approach, is out of the scope of this paper.

In Section 2, we give a description on the operation of IUO scheme. Section 3 summarizes the main assumption on the studied systems and defines the main processes that affect the performance of the system. The analytical model will be defined in Section 4 with which the performance analysis of a cell with IUO is carried out in Section 5. Some planning issues of the obtained results is summarized in Section 6 and the paper is concluded in Section 7.

\section{Intelligent Underlay-Overlay}

\subsection{Principle}

The IUO is a feature designed to allow a tighter frequency reuse for some of the available radio frequencies and therefore achieve a higher network capacity. It implements a two-layer network structure with a different reuse factor for each layer. The underlay adds capacity, the overlay provides coverage. To maintain optimum capacity, the base station assigns mobile traffic to either layer of the network according to actual interference levels. The IUO solution splits the available frequency spectrum into two bands. One consisting of frequencies that can only be used when a high $\mathrm{C} / \mathrm{I}$ ratio is ensured, the super frequencies, which are usu- ally used only near the Base Transceiver Station (BTS). The other band contains frequencies that can be used throughout the whole cell, the regular frequencies.

Every IUO cell has regular and super Transmitter Receivers (TRXs). Regular frequencies completely cover the cell. These frequencies can be reused by conventional criteria, using safe hand-over bounds to provide low probabilities for interference. Mobile stations are assigned to regular frequencies at the boundary where $\mathrm{C} / \mathrm{I}$ rate is under a specific level. Figure 1 shows the principle of the layer structure of IUO.

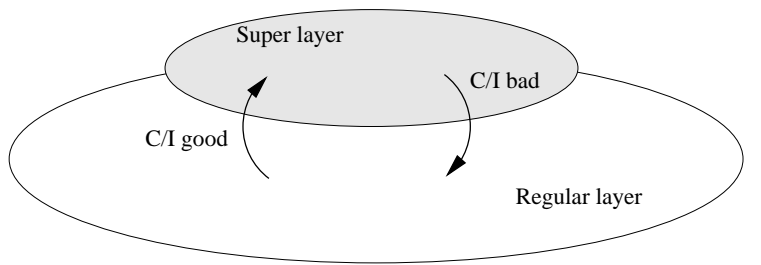

Figure 1. IUO principle

Super frequencies provide services in heavy traffic areas (downtown) of the cell, where $\mathrm{C} / \mathrm{I}$ rate is good (interference free area). Using different $\mathrm{C} / \mathrm{I}$ ratios, the coverage of the super layer (super frequencies) can be controlled. If IUO is combined with downlink power control, even better interference conditions can be maintained resulting in better call quality.

\subsection{Operation of IUO}

We assume the call admission operation of GSM network without IUO is known [12]. The IUO cell operation is described separately for standing (non-moving) and moving mobile stations (MS). The IUO algorithm does not distinguish moving or not moving MSs, this separation is done for a modeling assumption: we assume that a non-moving mobile's interference conditions will not change, and that the mobile will not try any handovers, unlike the moving mobile station.

- For both moving and non-moving mobile stations, the call request will first be served by a regular frequency, because the $\mathrm{C} / \mathrm{I}$ ratio of the call is not yet known. The $\mathrm{C} / \mathrm{I}$ ratio is calculated by comparing the downlink signal of the serving cell with the downlink signal of all neighboring cells, that use the same super frequencies.

- If the C/I value is better than a predefined "C/I good" threshold, then the connection will be served by a super frequency, otherwise it will remain at the regular layer. If this handover to the super frequency fails, because all super frequencies are occupied, then the connection will stay at the regular layer and try to get into the super layer after a specific time. 
- For the connections of non-moving MS, the call will be served at the same layer chosen according to the value of $\mathrm{C} / \mathrm{I}$ until its normal termination.

- For the connections of the moving MS, the value of C/I ratio may change after the setup of the connection. This implies a number of hand-over possibilities as follows:

1. For a connection going on a super frequency, the following hand-overs exist due to the movement of the MS:

$\diamond$ If the value of C/I ratio falls below the "C/I good" threshold, but there is another free super frequency where the $\mathrm{C} / \mathrm{I}$ ratio is appropriate, hand-over will be done, and the call will be kept on the super layer.

$\diamond$ If there is no free super frequency, or none of these has an appropriate $\mathrm{C} / \mathrm{I}$ ratio, the call gets back to regular layer, or if all the regular frequencies are occupied, the call will be lost.

2. For a connection going on a regular frequency, the following handovers exist due to the movement of the MS:

$\diamond$ If the $\mathrm{C} / \mathrm{I}$ value improves and reaches the " $\mathrm{C} / \mathrm{I}$ good" and there is a free super frequency, the connection will be moved to the super layer.

$\diamond$ An intra-cell hand-over to regular frequency of another TRX in the same cell.

$\diamond$ An inter-cell hand-over to regular frequency of another neighboring cell.

3 . The case of direct inter-cell hand-over from a super frequency to a neighboring cell is not considered in the model, because we assume that the C/I ratio is determined by the distance of the MS from the (closest) BTS. Hence, in our model, a super-regular hand-over always preceeds the inter-cell hand-over if the coverage factor of super layer is less than $100 \%$. In the applied framework it would also be possible to model direct inter-cell hand-over from super layer based on a probabilistic rule that describes the probability of direct inter-cell handover from super layer to a neighboring cell as a function of the coverage factor of super layer, but it is not considered in this paper.

Figure 2 summarizes all possible handovers in the IUO cell.

\section{Performance analysis}

\subsection{Modeling assumptions}

For the sake of building an analytical model of the system, we make the following assumptions:

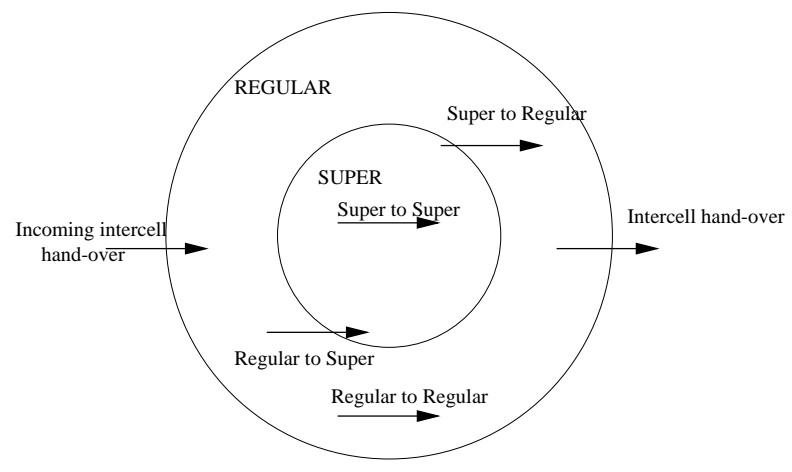

Figure 2. Possible handovers in the system

- Only one cell is considered in the analysis. All the interactions from the neighboring cells to the studied cell are taken into account as an aggregate incoming hand-over request process, which will first be served by regular frequencies.

- The studied cell implements IUO scheme with the super layer coverage factor cov of the cell area. Only one super frequency group is assumed, i.e., the same coverage factor is assumed for all super frequencies.

- The Base Transceiver Station of the cell manages $R C$ regular and $S C$ super frequencies. Thus, the total number of frequencies (channels) in the cell is $N=R C+S C$.

- The cell contains moving and non-moving mobile stations. The ratio of the moving MSs is $r_{m v}$.

- The moving MSs are assumed to move in a uniformly distributed random direction and with a constant speed $v_{M S}$.

- Super and regular frequencies of the same cell are not distinguished. The hand-over between regular frequencies or between super frequencies in the same cell are not taken into account, since it does not modify the number of busy frequencies of the layers.

- The different processes that control the events in the system are:

1. the new call arrival process is assumed to be a Poisson process with rate $\lambda$. We assume that the arrivals are uniformly distributed on the area of the cell and, therefore, cov of the calls will have $\mathrm{C} / \mathrm{I}$ value better than the " $\mathrm{C} / \mathrm{I}$ good" threshold.

2. the aggregate incoming hand-over is assumed to be a Poisson process with rate $\lambda_{h}$.

3. the time needed for the calculation of the $\mathrm{C} / \mathrm{I}$ parameter for a new connection is assumed to have an exponential distribution with mean of $1 / \mu_{\text {cal }}$. 
4. the call holding time, i.e., the time for normal call termination, is assumed to be exponentially distributed with average duration of $1 / \mu_{t}$.

5. The hand-over rate from super to regular frequencies and vice versa resulting from the movement of the moving MS are calculated taking into account: the speed of the MS $v_{M S}$, the radius of the cell, the coverage factor of the super layer, and the probability that the movement of the MS will lead to increase or decrease the value of $\mathrm{C} / \mathrm{I}$ ratio. According to this, we assume that the time needed for a moving MS to move from super to regular area or from regular to super area is an exponentially distributed random variable with mean value $1 / \mu_{\text {sup-reg }}$ or $1 / \mu_{\text {reg-sup }}$, respectively.

6. Similarly, we assume the channel dwell time to be an exponentially distributed random variable with mean value $1 / \mu_{\text {hout }}$, which is calculated with similar considerations to the previous point.

\subsection{The model}

With the above listed modeling assumptions the considered system (a cell) behaves as a Continuous Time Markov Chain. At any time instant the state of the cell is determined by the number of active calls of each class of frequencies, so we define it as the vector:

$$
X=\left(r_{r e g}, r_{s u p}, s u p\right)
$$

where

- $r_{r e g}$ is the number of connections served by regular frequencies and have $\mathrm{C} / \mathrm{I}<$ "C/I good",

- $r_{\text {sup }}$ is the number of connections with $\mathrm{C} / \mathrm{I} \geq$ "C/I good" that are served by regular frequencies, (there are two reasons for this situation: new calls with $\mathrm{C} / \mathrm{I} \geq$ "C/I good" are served by regular frequencies for the period of $\mathrm{C} / \mathrm{I}$ calculation; and calls with $\mathrm{C} / \mathrm{I} \geq$ "C/I good" are served by regular frequencies if all the super frequencies are occupied.)

- sup is the number of connections served by super frequencies.

Let $n_{r}(X)=r_{r e g}+r_{\text {sup }}$ and $n(X)$ denote the number of occupied regular and total frequencies in the cell, respectively. It is clear that a permissible state must satisfy the conditions $n(X) \leq N$ and $n_{r}(X) \leq R C$. Let $K$ denote the number of feasible states. Then, we can define $\Omega$ to denote the state space of the system given that the states are conveniently ordered from $0, \ldots, K-1$. The resulting model is thus homogeneous and irreducible on the finite state space $\Omega$ and therefore the steady state distribution $\mathbf{p}$ $=\left\{p_{x}\right\}, x=0 \ldots, K-1$, exists, unique and can be computed through the linear system of equations $\mathbf{p} \cdot \mathbf{Q}=\mathbf{0}$, and

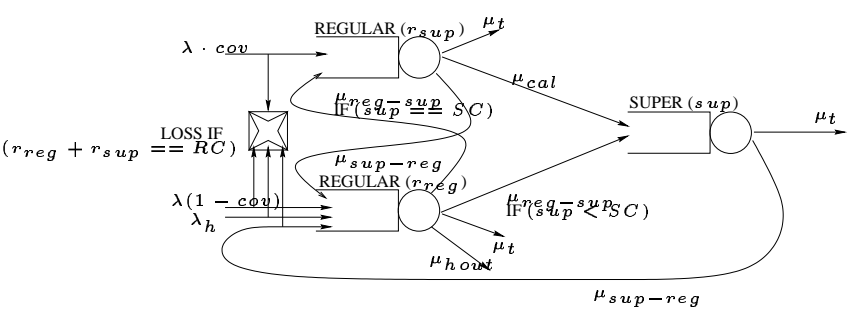

Figure 3. Queuing model of IUO cell

$\sum_{x=0}^{K-1} p_{x}=1$, where the matrix $\mathbf{Q}$ denotes the infinitesimal generator of the Markov chain.

The transition rates, the elements of matrix $\mathbf{Q}$, can be obtained from the analysis of the driving process in the system. We follow the style used in the rule definition syntax of the model specification language, MOSEL [13], which is based on a "Which state follows $\left(r_{r e g}, r_{s u p}, s u p\right)$ if..." logic. MOSEL is a model specification and evaluation language developed at the Department of Computer Science IV, University of Erlangen, Germany. Figure 3 shows the considered queuing model of the cell and Table 1 provides the rules that determine the transition rates following this style The Mosel description of the model is directly readable from this set of rules.

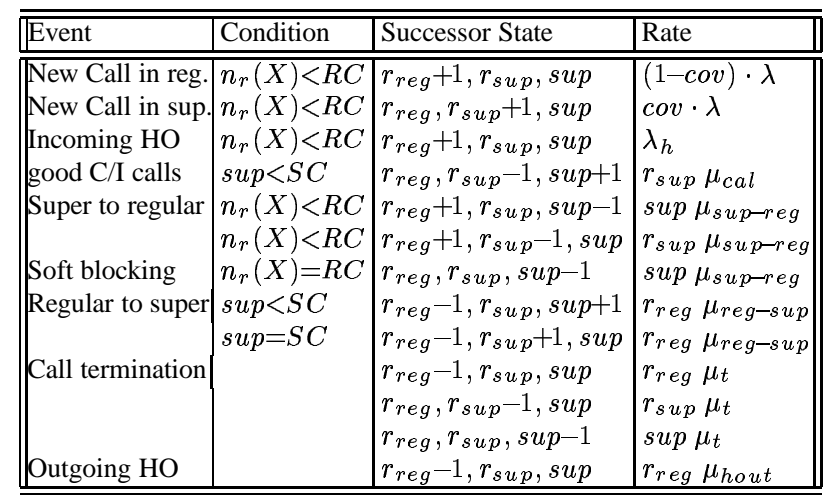

Table 1. Transition rules and rates

\begin{tabular}{||l|l||}
\hline \hline Parameter & Value \\
\hline \hline Number of regular frequencies, $R C$ & $28(4 \mathrm{TRX})$ \\
Number of super frequencies, $S C$ & $16(2 \mathrm{TRX})$ \\
Super area coverage factor, cov & $50 \%$ (var. of study) \\
Call holding time, $1 / \mu_{t}$ & $80 \mathrm{~s}$ (exponential) \\
Time for C/I calculation, $1 / \mu_{c a l}$ & $5 \mathrm{~s}$ (exponential) \\
Cell radius, $R$ & $3 \mathrm{~km}$ \\
Ratio of moving MS, $r_{m v}$ & $50 \%$ (var. of study) \\
Speed of moving MS, $v_{M S}$ & $50 \mathrm{~km} / \mathrm{h}$ (var. of study) \\
\hline \hline
\end{tabular}

Table 2. Default value of parameters

\section{Performance analysis of a cell with IUO}

In this section, we present some numerical results using the defined model. Table 2 summarizes the default values 


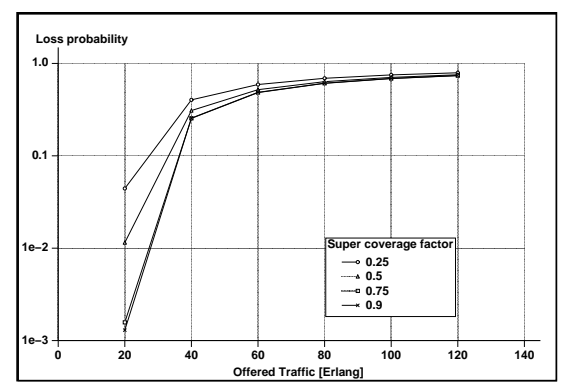

Figure 4. Loss probabilities versus super area coverage

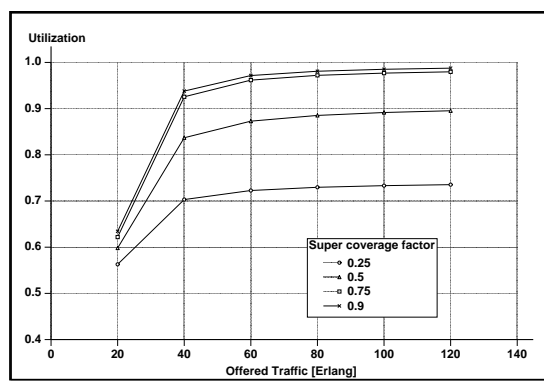

Figure 5. Utilization for different super area coverage

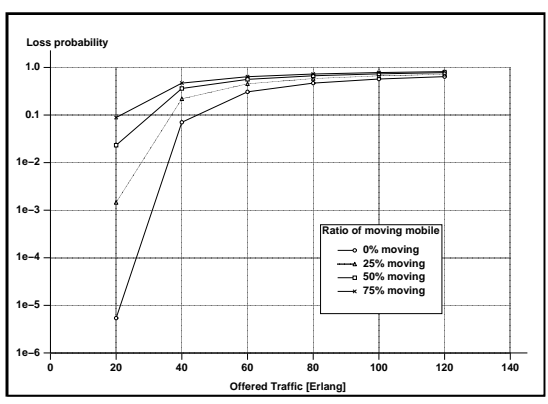

Figure 6. Loss probabilities versus moving MS ratio for the parameters used in the study. The use of different values will be explicitly mentioned. We present results on the call loss probability and the average utilization of channels (i.e., the carried traffic per channels) versus the offered traffic. Different results are provided by varying different parameters: the super area coverage factor, the number of $R C$ and $S C$ (but keep their sum constant, $N$ ), the ratio of moving MS, and the speed of moving MS. In all cases, the call loss probability is computed by summing the blocking probability of new calls and incoming handovers and the probability that a connection going on a super frequency will fail to be served due to the lack regular frequency when it's C/I value drops bellow "C/I good" threshold. In the model quality problems can only be due to interference problems indicated by leaving the super coverage area. This interference problem can lead to call drop, referring to soft blocking.

First, we study the effect of the super area coverage on the performance of the studied cell. Figure 4 shows the loss probability versus the offered traffic. The different curves refer to different super area coverage factor, cov. It can be seen that the higher cov results in lower loss probability. On the other hand, Fig. 5 shows that decreasing the area covered by the super layer has a negative impact on the utilization of the cell. This is in consistence with the conclusions obtained in [10]. This means that a trade-off or optimization should be done in the design phase of the network between the reduced utilization and increased loss probability versus the capacity increased by the higher reuse factor. It can be seen that the soft blocking has minor effect on the overall blocking rate even in case of high load situations.

In the rest of the results, we study the effect of movement on the performance of the IUO cell. Figures 6 and 7 show curves on the call loss probability and utilization. The different curves refer to different value of the ratio of moving MSs, $r_{m v}$. The results show that the higher ratio of movement results in higher loss probability which can be explained with the higher number of handovers resulting from the movement. The utilization point of view is shown in Figure 7. When the load is low then the movement helps in reaching better utilization in the cell, while in higher load conditions the increased movement has a negative effect on the utilization of the super frequencies which results in a reduced overall utilization of the cell. This is the reason for the crossing of the utilization curves between 20 and 60 Erlang offered traffic.

Figures 8 and 9 examine the effect of the speed of the moving MS given that $r_{m v}=25 \%$. Three values were chosen to reflect the movement in the downtown area of city; walking speed $(3 \mathrm{~km} / \mathrm{h})$, vehicle speed in residential area $(30 \mathrm{~km} / \mathrm{h})$, and vehicle speed in in-city main streets $(50 \mathrm{~km} / \mathrm{h})$. The results show that the increase of the speed implies very slight increment on the overall call loss probability. It is because the loss probability is composed by two main factors: the probability of rejecting new or incoming handover calls (referred to as blocking) and the soft blocking. Figure 8 depicts these factors separately. The overall loss probability practically coincides with the blocking probability. According to the expectations the soft blocking probability is significantly affected by the speed of moving MSs, but it has negligible effect on the overall loss since the blocking probability is at least an order of magnitude higher than the soft blocking probability. The decrease of the soft blocking probabilities from 80 Erlang offered load is due to the high blocking probability.

The results shows that the usage of IUO has a negative effect on the performance of one cell and, therefore, there should be an optimization for the gain of tighter reuse in the multiple cell network and the loss in the performance of each cell. The other conclusion is regarding the effect of the mobility of the performance of the IUO scheme. The results show that the IUO performs worse as mobility of the mobile stations increase.

\section{Planning issues}

To assist traffic dimensioning considerations additional computations were performed to evaluate the offered traffic with associated $2 \%$ loss. The input and output parameters 


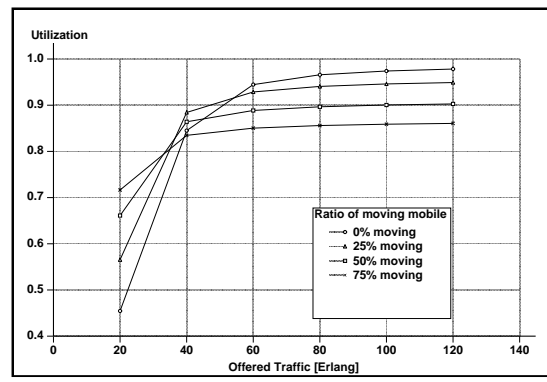

Figure 7. Utilization versus moving MS ratio

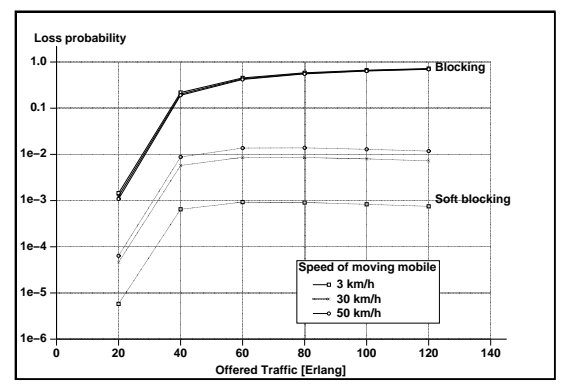

Figure 8. Loss probabilities versus moving MS speed

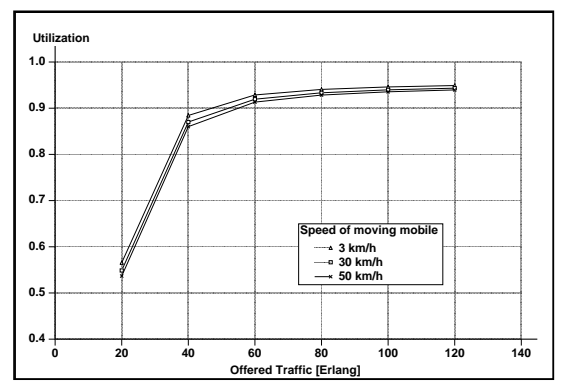

Figure 9. Utilization versus moving MS speed of the described numerical analysis are the offered traffic and the associated loss, respectively. Hence, to evaluate the offered traffic with associated $2 \%$ loss a numerical approximation is applied. We have evaluated the loss at 8, 16, 24, 32 , and 40 Erlang offered traffic and fit the obtained loss values by a 4th order polynome (through the Fit function of Mathematica [14]). The offered traffic with associated 2\% loss is calculated as the point where the polynomial equals to 0.02 . This approximation was found to be the best among those we tried (e.g. the fitting of the inverse of the loss function). The approximation was verified against the Erlang formulae. The error falls in the range of 0.5-1.2 Erlang.

In Figure 10 the capacity of various cell configurations deploying IUO with non-moving MSs is compared. The figure shows the offered traffic value (in Erlangs) versus the super coverage factor where the overall blocking probability is $2 \%$. The super coverage factor is ranging from $100 \%$ (the $\mathrm{C} / \mathrm{I}$ conditions are good throughout the coverage area of the cell) to $0 \%$ (only regular frequencies are used, this is practically the non-IUO configuration). The cell configurations differ in the number of regular and super TRXs, as shown in the legend. All the configurations are shown where 1 to 5 TRXs are used in the cell. For instance, with configuration $2+3$ at super coverage factor $50 \%$ maximum 15 Erlangs can be accepted with $2 \%$ blocking rate.

Several interesting conclusions can made reading the figure. As it is expected, the curve belonging to the configuration $\mathrm{A}+\mathrm{B}$ approximates the curve of configuration $\mathrm{A}+0$ (which is of course a horizontal line) as the super coverage factor approaches 0 . In the other end, A+B's curve approaches the curve of configuration $(A+B)+0$ as the coverage factor approaches $100 \%$. The difference between the various configurations' result stems from the assumptions that regular frequencies consist of 7 TCHs while super frequencies consist of $8 \mathrm{TCH}$ each, this also can be seen very well in the figure. For example, at $90 \%$ coverage factor the $3+2$ configuration (37 channels) outperforms the $4+1$ configuration (36 channels).

One the most useful outcomes of the paper is that decreasing the super coverage factor assuming a given cell configuration, does not mean immediate capacity degradation. For instance, for configuration $3+2$, the super coverage factor can be decreased down to $60 \%$ without degrading loosing capacity in the cell.

The same system is evaluated with $50 \%$ moving MSs. From Figure 11 it can be seen that the movement of MS decreases the performance of the cell. The main reason of this performance degradation is the increased need of the moving MSs for regular channels. Due to the high moving MSs ratio the regular channels become the bottleneck of the system and their availability characterize the system performance. The curve of the $4+1$ TRX cell configuration is slightly decreasing above 0.6 coverage factor. We think that this feature is not an evident property of cellular systems with IUO, but it results from the applied $\mu_{\text {sup-reg }}\left(v_{M S}, r_{m v}, \operatorname{cov}, R\right), \mu_{r e g-s u p}\left(v_{M S}, r_{m v}, \operatorname{cov}, R\right)$ functions:

$$
\begin{gathered}
\mu_{s u p-r e g}\left(v_{M S}, r_{m v}, \operatorname{cov}, R\right)=\frac{r_{m v} v_{M S}}{R \sqrt{2 c o v}}, \\
\mu_{r e g-s u p}\left(v_{M S}, r_{m v}, \operatorname{cov}, R\right)=\frac{r_{m v} v_{M S} \arcsin \frac{2 \sqrt{c o v}}{1+\sqrt{c o v}}}{\pi R\left(1-\frac{\sqrt{c o v}}{2}\right)} .
\end{gathered}
$$

These functions were found to be the best (regarding the overall model behaviour) to approximate the assumption that the MSs are uniformly distributed over the cell as well as their moving direction.

The practical use of the depicted results is as follows. Assumed the network planner has N TRXs in the given cell, he can easily compare the capacity of the different configurations of $\mathrm{N}+0$ (no IUO), (N-1)+1 (1 super frequency), (N2)+2 (2 super frequency), etc. and he can immediately read the allowable super coverage factor, which is a key factor for planning the reuse of the frequencies of the super frequency plane. Thus the planner can see the possible benefits of defining some of the TRXs as super frequencies. On the other hand if capacity extension is needed in the cell with $\mathrm{N}$ TRXs, the network planner can compare the solutions $(\mathrm{N}+1)+0, \mathrm{~N}+1$ or maybe $\mathrm{N}+2$, where the last two cases allow the planner to keep the original frequencies in the regu- 


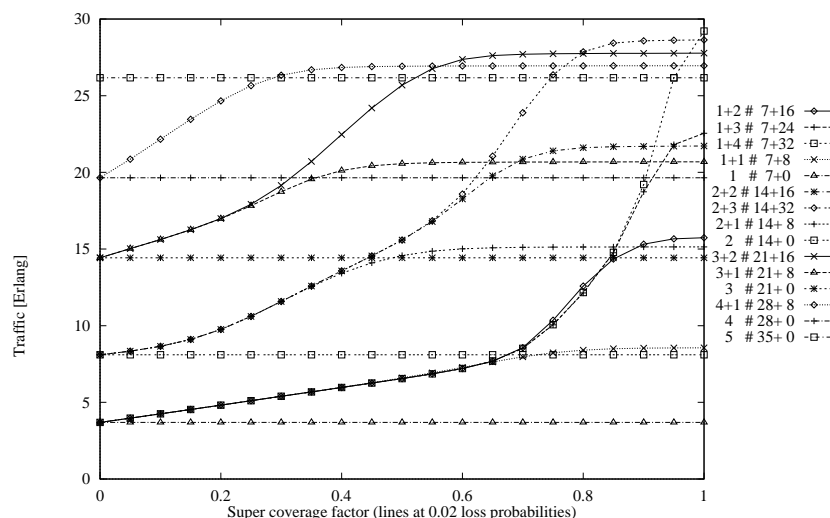

Figure 10. Performance of different cell configurations with non-moving MSs

lar TRXs and finding new frequencies for the super TRXs, which frequencies may suffer interference, handled by IUO.

\section{Conclusions}

The paper presented an analytical model for GSM-based cellular mobile network that implements the Intelligent Underlay-Overlay scheme to increase the frequency reuse and, therefore, the capacity of the network. The Markov analysis based investigations has focused on the effect of using this scheme on the one-cell performance taking into account the coverage factor of the super frequencies and the movement of the mobile stations.

The analysis results characterize the effect of IUO on the performance of a cell as a function of the coverage factor, the mobility of MSs, and their speed. It is also discussed how the obtained results can be used in practical mobile network planning.

Further research is planned to include several super frequency groups into the model, as well as studying the effect when the MSs do not have to camp first on the regular layer for a while before accommodated into the super layer, as Nokia proposed recently.

\section{References} [1] Nokia Telecommunications Web Site:
www.nokia.com.

[2] R. Steele, M. Nofal, Teletrafic Performance of Microcellular Personal Communication Networks, IEEE Proceedings-I Vol. 139, No. 4, August 1992.

[3] S. Marano, C. Mastroianni, A Hierarchical Network Scheme for Multi-layered Cellular Systems, Proc. of the VTC 1997 Phoenix, Arizona, USA, May 1997.

[4] P.E. Mogensen, J. Wigard, F. Frederiksen, Performance of Slow Frequency Hopping in GSM, COST 231, Sept. 1995.

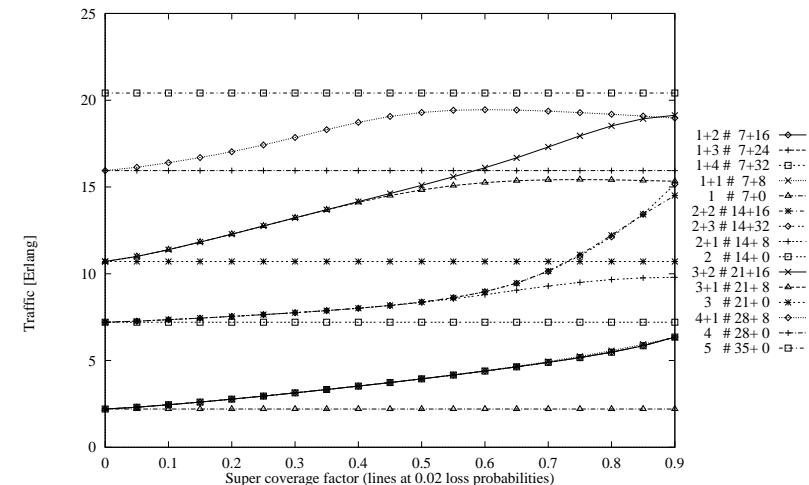

Figure 11. Performance of different cell configurations with moving MSs

[5] M. Frodigh, Reuse partioning combined with traffic adaptive channel assignment for highway microcellular systems Globecom '92

[6] S. Papavassiliou, L. Tassiulas, P. Tandon, Meeting QoS requirements in cellular network with reuse partitioning IEEE Journal of selected areas in Communications, 1994

[7] J. Zander, Generalized reuse partitioning in cellular mobile radio, Proc. Veh Tech Conf, 1993

[8] Y. Ling, J. Elling, T. Toftegrad Nielson, Capacity of Intelligent Underlay and Overlay Network, Proc. of the IEE Colloquium on Advanced TDMA Techniques and Applications, Oct. 1996, pp. 23-31.

[9] V. Géróné Molnár, Analysis of Capacity Enhancement Techniques in GSM Networks: Intelligent Underlay Overlay, M.Sc. thesis, Technical University of Budapest, 1998., in Hungarian

[10] T. T. Nielsen, J. Wigard, P. Morgensen, On The Capacity of a GSM Frequency Hopping Network with Intelligent Underlay-Overlay, Proc. of the VTC 1997 Phoenix, Arizona, USA, May 1997.

[11] J. Wigard, T. T. Nielsen, P. H. Michaelsen, P. Morgensen, Improved Intelligent Underlay-Overlay combined with Frequency Hopping in GSM, PIMRC 1997, Helsinki, Finland.

[12] GSM Recommendations.

[13] G. Bolch, H. Herold, MOSEL: Modeling, Specification, and Evaluation Language. Technical Report TRi4-95-02, University of Erlangen-Nürnberg, 1996.

[14] S. Wolfram, Mathematica, Addison-Wesley 1991. 